\title{
Síntese e Caracterização de Copolímeros de Estireno e Divinilbenzeno Clorometilados
}

\author{
Viviane G. Teixeira \\ Departamento de Química, UFRRJ e IMA, UFRJ \\ Fernanda M. B. Coutinho \\ Instituto de Química, UERJ e IMA, UFRJ \\ Ailton S. Gomes \\ IMA, UFRJ
}

\begin{abstract}
Resumo: A reação de clorometilação de copolímeros de estireno e divinilbenzeno (S-DVB) em presença de HCl, formaldeído e cloreto de zinco foi estudada em meios aquoso e orgânico. O copolímero S-DVB foi, primeiramente, sintetizado por polimerização em suspensão e caracterizado quanto às suas propriedades porosas. Em meio aquoso, o copolímero foi clorometilado borbulhando-se $\mathrm{HCl}$ na mistura de solução aquosa de formaldeído a 37\% (formalina 37\%) e solução aquosa de cloreto de zinco (catalisador). Em meio orgânico (1,2-dicloroetano), foram usados $\mathrm{HCl}$ (borbulhado na solução reacional), paraformaldeído e cloreto de zinco sólido. A presença de grupos clorometila foi identificada por espectroscopia na região do infravermelho e o teor desses grupos foi determinado por titulação potenciométrica dos íons cloreto, liberados após a reação de quaternização com piridina. A reação em meio aquoso originou copolímeros com baixos graus de clorometilação, mesmo sob condições drásticas (uso do catalisador sólido e ácido clorídrico concentrado como meio reacional). Em meio orgânico, devido à maior estabilidade do eletrófilo, foram originados copolímeros com teores mais altos de grupos clorometila. Foi estudada a influência dos parâmetros reacionais sobre o grau de clorometilação dos copolímeros em meio orgânico. A temperatura e a razão copolímero/catalisador foram parâmetros determinantes do grau de clorometilação obtido.
\end{abstract}

Palavras-chave: Clorometilação, copolímeros de estireno e divinilbenzeno, ácido clorídrico, formaldeído, cloreto de zinco.

\section{Synthesis and Characterization of Chloromethylated Styrene-Divinylbenzene Copolymers}

Abstract: Cloromethylation of styrene-divinylbenzene copolymer (S-DVB) in presence of hydrogen chloride, formaldehyde and zinc chloride was studied in aqueous and organic media. S-DVB was first synthesized by suspension polymerization and its porosity was characterized. In aqueous medium, S-DVB was chloromethylated by using a mixture of hydrogen chloride, aqueous formaldehyde solution (formalin 37\%) and aqueous zinc chloride solution as catalyst. In organic medium (1,2-dichloroethane), a mixture of hydrogen chloride, paraformaldehyde and solid zinc chloride was employed. The presence of chloromethyl groups in the copolymer was investigated by infrared spectroscopy analysis and titration of chloride ions, after a quaternization reaction between the chloromethylated copolymer and pyridine. The reaction in aqueous medium seems to be very difficult to occur even in the presence of solid zinc chloride (catalyst) and concentrated hydrochloric acid as reaction medium. Organic medium offers a better condition for aromatic eletrophilic substitution, where the reaction occurred in a more satisfactory way. The influence of reaction parameters was studied. Temperature and copolymer/catalyst ratio were the main responsible factors for the attained chloromethylation degree.

Keywords: Chloromethylation, styrene-divinylbenzene copolymers, hydrogen chloride, formaldehyde, zinc chloride.

\section{Introdução}

A separação de espécies metálicas por métodos baseados em equilíbrios sólido-líquido tem sido alvo de um grande número de estudos, incluindo-se aí o uso de resinas quelantes como suportes para a cromatografia de extração. Este método tem demonstrado ser mais seletivo do que a cromatografia de troca iônica, ganhando espaço significativo no campo da separação de espécies metálicas ${ }^{[1]}$. Resinas quelantes também têm se mostrado importantes para a síntese de catalisadores heterogeneizados. Nesses sistemas, a imobilização dos catalisadores metálicos, comumente usados em reações homegêneas, é feita através da sua complexação com a resina ${ }^{[2]}$.

Autor para correspondência: Fernanda M. B. Coutinho, Instituto de Macromoléculas Professora Eloisa Mano, UFRJ, Caixa Postal 68525, CEP:21945-970, Rio de Janeiro, RJ. E-mail: fern@ima.ufrj.br 
As resinas quelantes se caracterizam pela presença de um grupo complexante imobilizado em um suporte insolúvel. Os materiais sintéticos mais usados como suportes para a imobilização de grupos quelantes são os copolímeros de estireno (S) e divinilbenzeno (DVB) obtidos por polimerização em suspensão iniciada via radicais livres. Essa técnica é preferida principalmente quando o uso final do material é a cromatografia iônica, pois permite o controle do tamanho de partícula e da porosidade do suporte, parâmetros determinantes na otimização do processo cromatográfico ${ }^{[1,3]}$.

As rotas sintéticas usadas para imobilizar um grupo complexante em copolímeros S-DVB são as mais variadas e dependem do tipo de grupo de interesse. Contudo, a maioria das rotas é facilitada por uma reação prévia de clorometilação dos anéis aromáticos do suporte (alquilação de Friedel-Crafts), tornando-o mais reativo pela presença dos átomos de cloro, bastante lábeis, e por isso mesmo, facilmente substituíveis em reações subseqüentes. A clorometilação desses materiais é classicamente conseguida com o uso de éter clorometílico ou éter bisclorometílico (substâncias altamente carcinogênicas) em presença de um ácido de Lewis como catalisador. Muitos estudos têm sido então propostos com o intuito de estabelecer rotas de síntese mais seguras, que não utilizem esses reagentes ${ }^{[4,5]}$.

Com esse objetivo, a geração do éter clorometílico in situ foi proposta como alternativa à manipulação direta desse agente clorometilante ${ }^{[5]}$. Éteres alquilclorometílicos de cadeias longas também foram usados como agentes clorometilantes, já que são menos insalubres, devido à sua menor volatilidade ${ }^{[6]}$. Todavia, rotas envolvendo outros tipos de agentes clorometilantes têm sido mais estudadas. Compostos como 1-cloro-4clorometoxibutano foram usados na clorometilação de benzeno e de derivados do tolueno em presença de $\mathrm{SnCl}_{4}$ e mostraramse bastante efetivos ${ }^{[7,8]}$. DeHaan e colaboradores estudaram a cinética da reação de clorometilação de benzeno e tolueno empregando cloreto de metoxiacetila e $\mathrm{AlCl}_{3}{ }^{[9]}$. Muitas rotas baseiam-se no uso de formaldeído e um composto capaz de gerar íons cloreto. Nessas reações, formaldeído é gerado in situ a partir de paraformaldeído ou trioxano e compostos como cloreto de trimetil-silano e $\mathrm{HCl}$ gasoso têm sido usados como fonte de íons cloreto, normalmente em presença de anidrido acético. A adição nucleofílica dos íons cloreto à carbonila aldeídica leva a um intermediário que, em presença de um ácido de Lewis, forma o eletrófilo capaz de atacar o anel aromático. $\mathrm{O}$ uso de $\mathrm{AlCl}_{3}$ e $\mathrm{SnCl}_{4}$ como catalisadores levam a maiores graus de clorometilação. Entretanto, a extensão da reação deve ser bem controlada, pois esses catalisadores produzem reticulações adicionais por meio de pontes metilênicas, geradas a partir da reação de substituição eletrofílica entre um grupo clorometila já imobilizado e um anel aromático livre. Um controle mais fácil da reação é conseguido pelo uso de $\mathrm{ZnCl}_{2}$, um ácido de Lewis mais fraco ${ }^{[10-14]}$.

Não só o tipo de catalisador leva ao controle da clorometilação, mas também parâmetros como temperatura, tempo de reação e quantidade de catalisador podem definir o grau de clorometilação dos copolímeros S-DVB. Entretanto, esses fatores não determinam a distribuição dos grupos clorometila no copolímero. Essa característica é normalmente determinada pelo acesso do meio reacional à rede polimérica, que pode ser controlado pela afinidade entre o solvente usado como meio reacional e o substrato ${ }^{[15]}$.

Uma outra forma de se obter materiais contendo grupos clorometila é a utilização de um terceiro monômero já clorometilado na reação de polimerização, além de S e DVB. Essa opção porém, não é usual devido ao alto custo do clorometilestireno (CMS) comparado ao custo total de se produzir o copolímero de S-DVB e clorometilá-lo posteriormente ${ }^{[16]}$. Um outro ponto positivo da reação de clorometilação posterior é o de oferecer a possibilidade de se usar resinas comerciais por certos grupos de pesquisa que desejam apenas estudar a modificação do material pronto. Além disso, a síntese de copolímeros de S-DVB com porosidade controlada é de amplo domínio. A inserção do terceiro monômero na síntese leva a diferenças na porosidade do material, necessitando de maiores cuidados. Em relação à distribuição dos grupos, e talvez esse seja o principal motivo, é muito mais fácil controlar o curso da reação de clorometilação para que se dê preferencialmente nas regiões mais acessíveis do material do que controlar a reação de polimerização em suspensão entre os três monômeros, S, DVB e CMS, a ponto de que os grupos clorometila sejam bastante acessíveis. Como a reação de clorometilação é feita, normalmente, com o objetivo de se substituir o átomo de cloro por meio de reações subseqüentes, a acessibilidade do meio reacional a esses grupos é fundamental.

Neste trabalho, então, propõe-se a clorometilação de copolímeros $\mathrm{S}-\mathrm{DVB}$ usando-se a mistura $\mathrm{HCl} /$ formaldeído como agente clorometilante em presença de $\mathrm{ZnCl}_{2}$ (catalisador), em meios aquoso e orgânico. O estudo em diferentes meios visa relacionar a porosidade do copolímero e o acesso do meio reacional à estrutura polimérica, podendo-se assim controlar a extensão da clorometilação e a distribuição dos grupos nessa estrutura.

\section{Experimental}

\section{Reagentes}

Estireno (S) e divinilbenzeno (DVB) (cedidos pela Nitriflex S.A.) foram lavados com solução aquosa de $\mathrm{NaOH}$ a $5 \%(\mathrm{~m} / \mathrm{v})$ seguida de água para eliminação do inibidor. 2,2'-Azobisisobutironitrila (AIBN) (cedido pela Metacril S.A.) foi recristalizado em metanol. Os demais reagentes utilizados apresentavam grau de pureza PA e foram usados tal como recebidos.

\section{Instrumental}

Para determinação da área específica do copolímero foi usado o instrumento ASAP mod. 2010, Micromeritics. A distribuição de tamanhos de poros foi determinada em porosímetro de mercúrio Micropore Autoscan 33. As titulações potenciométricas foram conduzidas utilizando-se um eletrodo de oxidação-redução combinado contendo anel de prata acoplado a um potenciômetro Analyser . A presença de grupos clorometila nos copolímeros foi identificada por 
espectroscopia na região do infravermelho em um espectrofotômetro Perkin Elmer 1720X

\section{Síntese do copolímero S-DVB}

O suporte foi sintetizado pela copolimerização em suspensão de S e DVB, usando-se AIBN como iniciador (1\% molar em relação ao total de monômeros) e n-heptano como diluente da mistura de monomérica (S/DVB) na proporção volumétrica de $1 / 1$. Na fase aquosa, poli(álcool vinílico) a $5 \%$ $(\mathrm{m} / \mathrm{v})$ (grau de hidrólise $88 \%$ ) foi usado como agente de suspensão e $\mathrm{NaCl}$ a $5 \%(\mathrm{~m} / \mathrm{v})$, para diminuir a solubilidade dos monômeros em água. A razão volumétrica entre a fase aquosa e a fase orgânica foi de 4/1. O copolímero obtido foi lavado com água a $80^{\circ} \mathrm{C}$ e peneirado. A fração de interesse $(45$ a 80 mesh) foi lavada com etanol, metanol e, finalmente, seca em estufa.

\section{Caracterização da porosidade do copolímero S-DVB}

Para a caracterização do suporte quanto à sua porosidade foram avaliados os seguintes parâmetros: densidade aparente (modificação do método ASTM D1895); volume de poros fixos por retenção de água; inchamento percentual em tolueno e em n-heptano; área específica por adsorção de nitrogênio segundo o método de BET; distribuição de tamanhos de poro por porosimetria de intrusão de mercúrio.

\section{Reação de clorometilação do copolímero de S-DVB}

A clorometilação foi realizada em meio aquoso e em meio orgânico.

\section{Reação em meio aquoso}

Após tratamento com metanol seguido de água deionizada, $1 \mathrm{~g}$ de copolímero úmido foi transferido para um balão tritubulado ao qual foram também adicionadas quantidades variáveis de água ou de ácido clorídrico concentrado, formalina $37 \%$ e solução aquosa de $\mathrm{ZnCl}_{2}(1,36 \mathrm{M})$. Foram acoplados ao balão um gerador de $\mathrm{HCl}$ gasoso $[\mathrm{HCl}(\mathrm{g})]$ e um condensador de refluxo acoplado a um coletor de $\mathrm{HCl}(\mathrm{g})$ contendo $\mathrm{NaOH}$ sólido (sistema de proteção). O sistema reacional foi homogeneizado com agitador magnético e aquecido com auxílio de banho termostatizado.

\section{Reação em meio orgânico}

Transferiu-se $1 \mathrm{~g}$ de copolímero seco para o balão tritubulado ao qual também foram adicionadas quantidades variáveis de 1,2-dicloroetano (1,2-DCE), paraformaldeído e $\mathrm{ZnCl}_{2}$ sólido. Foi utilizado o mesmo aparato descrito para a reação em meio aquoso.

\section{Purificação dos copolímeros S-DVB clorometilados}

Os copolímeros clorometilados em meio aquoso foram lavados com água deionizada até neutralidade e ausência de íons cloreto, testada com $\mathrm{AgNO}_{3}$. Já os copolímeros clorometilados em meio orgânico foram primeiramente lavados com etanol e posteriormente com mistura água/etanol (1/1) até neutralização e ausência de íons cloreto. Nos dois casos, os copolímeros foram posteriormente lavados com etanol e secos em estufa.

\section{Determinação do teor de grupos clorometila nos copolímeros $S$-DVB clorometilados}

A determinação quantitativa de grupos clorometila incorporados no copolímero S-DVB envolveu a reação de quaternização da piridina com o copolímero clorometilado (S-DVB- $\mathrm{CH}_{2} \mathrm{Cl}$ ) seguida de tratamento com N,N-dimetilformamida e $\mathrm{HNO}_{3}$ concentrado. Os íons cloreto liberados para a solução foram titulados potenciometricamente com solução padrão de $\mathrm{AgNO}_{3}$. O resultado foi expresso em número de mmoles de grupos $-\mathrm{CH}_{2} \mathrm{Cl}$ por grama de copolímero.

\section{Espectroscopia na região do infravermelho (IV)}

As amostras foram diluídas com $\mathrm{KBr}(1 \%)$ e preparadas sob a forma de pastilhas. A presença de absorção na freqüência de $1265 \mathrm{~cm}^{-1}$ (deformação axial da ligação $\mathrm{C}$-Cl) identificou a presença de grupos $\mathrm{CH}_{2} \mathrm{Cl}$ nos copolímeros.

\section{Resultados e Discussão}

\section{Características Porosas do Copolímero S-DVB}

Na Tabela 1 são apresentadas as características porosas do copolímero S-DVB sintetizado. O baixo valor de densidade aparente e o alto volume de poros fixos mostram que o material é bastante poroso. A área de $127 \mathrm{~m}^{2} \mathrm{~g}^{-1}$, apesar de não ser muito alta, está associada a poros com tamanhos na faixa de 500 a $1000 \AA$, o que traduz uma estrutura porosa de fácil acesso à solução reagente. Os valores próximos para o grau de inchamento da matriz polimérica em tolueno e em n-heptano mostram que apenas uma fração pequena de fase gel está presente no copolímero. A porosidade acessível ao meio reacional na etapa de clorometilação deverá ser, basicamente, a porosidade permanente. Portanto, a reação deverá ocorrer, principalmente, na superfície dos poros fixos e, em menor extensão, nos poros expandidos pelo solvente usado na reação. Este copolímero foi usado no estudo da clorometilação tanto em meio aquoso como em meio orgânico.

\section{Estudo da clorometilação do copolímero S-DVB em meio aquoso}

Na grande maioria dos casos, as resinas quelantes são usadas para separação de espécies metálicas presentes em solução aquosa ou para remediação de rejeitos aquosos. Portanto, o acesso das espécies iônicas aos sítios quelantes da resina é limitado pela capacidade de difusão da solução aquosa pela rede polimérica. A escolha da água como meio para a reação

Tabela 1. Características porosas do copolímero S-DVB

\begin{tabular}{ccccccc}
\hline Parâmetro & $\begin{array}{c}\mathbf{d}_{\mathrm{a}} \\
\left(\mathbf{g ~ c m}^{-3}\right)\end{array}$ & $\begin{array}{c}\mathbf{V}_{\mathrm{pf}} \\
\left(\mathbf{c m}^{\mathbf{3}} \mathbf{g}^{-1}\right)\end{array}$ & $\begin{array}{c}\mathbf{A} \\
\left(\mathbf{m}^{2} \mathbf{g}^{-1}\right)\end{array}$ & $\begin{array}{c}\mathbf{D} \\
(\AA)\end{array}$ & $\begin{array}{c}\mathbf{I}_{\text {tol }} \\
(\boldsymbol{\%})\end{array}$ & $\begin{array}{c}\mathbf{I}_{\text {hep }} \\
(\boldsymbol{\%})\end{array}$ \\
\hline Valor & 0,30 & 1,06 & 127 & $500-1000$ & 24 & 16 \\
\hline
\end{tabular}

$\mathrm{d}_{\mathrm{a}}=$ densidade aparente; $\mathrm{V}_{\mathrm{pf}}=$ volume de poros fixos; $\mathrm{A}=$ área específica $\mathrm{D}^{\mathrm{a}}=$ diâmetros de poro na faixa de maior concentração; $\mathrm{I}_{\text {tol }}=$ inchamento percentual em tolueno; $\mathrm{I}_{\text {hep }}=$ inchamento percentual em n-heptano. 
Tabela 2. Condições reacionais e resultados para a reação de clorometilação de S-DVB em meio aquoso.

\begin{tabular}{|c|c|c|c|c|c|c|c|}
\hline Reação & IV $V_{1265 \mathrm{~cm}^{-1}}$ & $\begin{array}{c}\mathrm{CH}_{2} \mathrm{Cl} \\
\left(\mathrm{mmol} \mathrm{g}^{-1}\right)\end{array}$ & $\begin{array}{c}\mathbf{V}_{\text {formalina }} \\
\text { (mL) }\end{array}$ & $\begin{array}{c}\mathbf{V}_{\mathrm{ZnCl2}(1,36 \mathrm{M})} \\
\quad(\mathrm{mL})\end{array}$ & $\begin{array}{c}\mathrm{V}_{\mathrm{H} 2 \mathrm{O} \text { ou } \mathrm{HCl}} \\
(\mathrm{mL})\end{array}$ & $\begin{array}{c}t_{\text {reacão }} \\
\text { (h) }\end{array}$ & $\begin{array}{c}\mathbf{T} \\
\left({ }^{\circ} \mathbf{C}\right)\end{array}$ \\
\hline CMA1 & $(+)$ & 0,0648 & 10 & \multirow{4}{*}{2} & \multirow{8}{*}{$60 * *$} & \multirow{7}{*}{3} & \multirow{8}{*}{60} \\
\hline CMA2 & $(+)$ & 0,0813 & 5 & & & & \\
\hline CMA3 & $(+)$ & 0,0488 & 2,5 & & & & \\
\hline CMA4 & $(-)$ & 0,0224 & 1 & & & & \\
\hline CMA5 & $(+)$ & nd & \multirow{4}{*}{5} & 3 & & & \\
\hline CMA6 & $(-)$ & nd & & 1 & & & \\
\hline CMA7 & $(-)$ & nd & & 0,5 & & & \\
\hline CMA8 & $(-)$ & nd & & 2 & & 1 & \\
\hline CMA9 & $(+)$ & nd & \multirow{2}{*}{40} & \multirow{2}{*}{$1 g^{*}$} & $15 * * *$ & \multirow{4}{*}{3} & 75 \\
\hline CMA10 & $(-)$ & nd & & & $20 * * *$ & & 95 \\
\hline CMA11 & $(+)$ & 0,265 & \multirow{2}{*}{30} & 2 & $30 * * *$ & & \multirow{2}{*}{60} \\
\hline CMA12 & $(+)$ & 0,268 & & $0,25 \mathrm{~g} *$ & $15 * * *$ & & \\
\hline
\end{tabular}

IV1265 $\mathrm{cm}^{-1}=$ presença de absorção em $1265 \mathrm{~cm}^{-1} ;$ nd $=$ não determinado; $* \mathrm{ZnCl}_{2}$ sólido; ${ }^{*} \mathrm{HCl}$ foi borbulhado na mistura aquosa durante o período reacional; *** $\mathrm{HCl}$ concentrado.

de clorometilação de copolímeros S-DVB visa proporcionar uma melhor distribuição dos grupos na parte do copolímero acessível a esse meio, já que os grupos presentes em regiões inacessíveis à água não contribuirão para a capacidade de complexação da resina.

A reação de clorometilação em meio aquoso foi conduzida com formalina (solução aquosa de formaldeído a 37\%), borbulhamento constante de $\mathrm{HCl}(\mathrm{g})$ e solução de $\mathrm{ZnCl}_{2}(1,36$ M). Esta reação foi descrita por Fuson e Mckeever ${ }^{[16]}$, segundo o método de Blanc para a síntese do cloreto de benzila. Os autores citam que a clorometilação de benzeno pode ser conseguida, usando formalina em substituição ao paraformaldeído, porém quantidades maiores de catalisador devem ser adicionadas. A Tabela 2 resume as condições empregadas para a reação de clorometilação em meio aquoso, assim como os teores de grupos clorometila incorporados no copolímero S-DVB.

Neste estudo foi, primeiramente, variada a quantidade de formalina usada, mantendo-se todos os demais parâmetros constantes. Entretanto, os graus de clorometilação alcançados foram muito baixos. A espectroscopia na região de infravermelho confirmou a incorporação do grupo clorometila pela presença de absorção em $1265 \mathrm{~cm}^{-1}$. Esse resultado descarta a possibilidade do grau de clorometilação obtido por meio da titulação potenciométrica dever-se à contaminação por íons cloreto do meio reacional (Figura 1).

Como não houve um aumento do grau de clorometilação com o aumento da quantidade de formalina de 5 para $10 \mathrm{~mL}$, foi estudado o efeito da variação da quantidade de catalisador, mantendo-se o volume de formalina em $5 \mathrm{~mL}$. Entretanto, também não houve aumento significativo do grau de clorometilação com aumento da quantidade de catalisador (reação CMA5). O espectro de infravermelho do copolímero
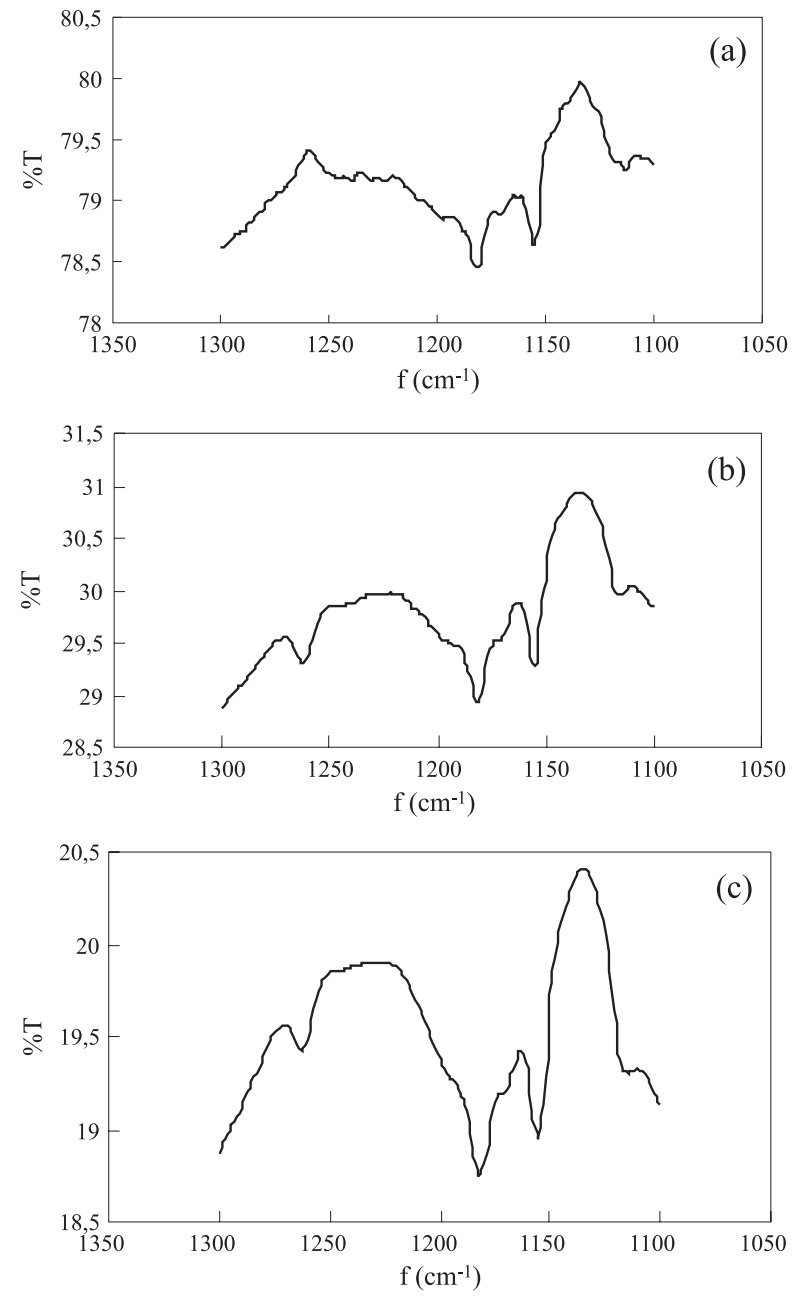

Figura 1. Espectros de absorção na região do infravermelho do copolímero de partida (a), do copolímero clorometilado em CMA2 (b) e em CMA5 (c) 
Tabela 3. Condições reacionais e resultados para as reações preliminares de clorometilação de S-DVB em meio orgânico.

\begin{tabular}{|c|c|c|c|c|c|}
\hline Reação & $\mathrm{IV}_{1265 \mathrm{~cm}^{-1}}$ & $\begin{array}{c}\mathrm{CH}_{2} \mathrm{Cl} \\
\left(\mathrm{mmol} \mathrm{g}^{-1}\right)\end{array}$ & $\underset{\text { (g) }}{\text { Massa de } \mathrm{ZnCl}_{2}}$ & $\begin{array}{c}\mathbf{t}_{\text {reação }} / \mathbf{t} \mathbf{H C l} \\
\text { (h) }\end{array}$ & $\begin{array}{c}\mathbf{T} \\
\left({ }^{\circ} \mathbf{C}\right)\end{array}$ \\
\hline CMO1 & $(+)$ & 0,237 & $2 \mathrm{~mL}^{*}$ & \multirow{3}{*}{$3 / 3$} & 60 \\
\hline $\mathrm{CMO} 2$ & $(+)$ & 0,850 & \multirow{4}{*}{0,25} & & 75 \\
\hline CMO3 & $(+)$ & 0,378 & & & 60 \\
\hline $\mathrm{CMO} 4$ & $(+)$ & 0,610 & & $5,5 / 5,5$ & \multirow{2}{*}{75} \\
\hline CMO5 & $(+)$ & 0,616 & & $5,5 / 2$ & \\
\hline
\end{tabular}

Massa de paraformaldeído $=0,5 \mathrm{~g}$; volume de 1,2-DCE $=20 \mathrm{~mL} ;$ * solução de $\mathrm{ZnCl}_{2}(0,92 \mathrm{M}) \mathrm{em} \mathrm{THF;} \mathrm{t}_{\text {reaçã }} / \mathrm{t}_{\mathrm{HCl}}=$ tempo de reação/tempo de borbulhamento de $\mathrm{HCl}$.

clorometilado na reação CMA5 apresentou bandas com intensidades próximas às dos espectros da série de reações anterior. Por isso, o teor exato de grupos clorometila não foi determinado. $\mathrm{O}$ uso de quantidades mais baixas de catalisador não levou à clorometilação do S-DVB.

Com o objetivo de aumentar o grau de clorometilação, em meio aquoso, dos copolímeros, foram feitas reações em condições extremas, usando-se volumes de formalina altos, catalisador sólido, temperaturas altas e substituindo-se água por $\mathrm{HCl}$ concentrado (reações CMA9 a CMA12). Entretanto, os graus de clorometilação obtidos nessas reações não seguiram um perfil de comportamento. Apesar de se obter graus de clorometilação maiores, esses resultados não são suficientemente satisfatórios para justificar e compensar o uso de condições tão severas.

A dificuldade em se promover a reação de clorometilação em meio aquoso pode estar associdada à baixa estabilidade do eletrófilo nesse meio. Mesmo a manipulação dos parâmetros responsáveis pelo deslocamento do equilíbrio da reação de clorometilação não foram suficientes para compensar a instabilidade do eletrófilo. Portanto, a reação em meio aquoso se mostrou inadequada para atingir graus de clorometilação satisfatórios.

\section{Estudo da clorometilação do copolímero S-DVB em meio orgânico}

A mesma reação de clorometilação foi conduzida em meio orgânico. 1,2-Dicloroetano foi usado como solvente por apresentar afinidade pelo copolímero, o que leva à funcionalização da parte gel do material. A importância dessa característica do solvente deve-se à expectativa de se avaliar a extensão da clorometilação na rede polimérica e associá-la às reações posteriores de inserção de grupos complexantes.

Paraformaldeído foi usado como fonte de formaldeído no meio reacional. Inicialmente, foram feitas algumas reações preliminares, conforme mostra a Tabela 3. O catalisador foi usado na forma sólida ou como solução em THF. Observouse um maior grau de clorometilação quando o $\mathrm{ZnCl}_{2}$ sólido foi utilizado (CMO1 e CMO2). Isto se deve ao efeito de complexação do THF (base de Lewis) com o $\mathrm{ZnCl}_{2}$ (ácido de Lewis), diminuindo sua atividade catalítica. Sendo assim, aptou-se pelo uso do sólido, embora o emprego da solução permita uma melhor reprodutibilidade da quantidade de catalisador utilizada nas diversas reações. A temperatura também foi variada. A $75^{\circ} \mathrm{C}$ obteve-se um grau de clorometilação mais alto do que a $60^{\circ} \mathrm{C}$. Foram ainda variados o tempo de reação e o tempo de borbulhamento de $\mathrm{HCl}$. Não foi observado um aumento do grau de clorometilação com o aumento do tempo reacional de $3 \mathrm{~h}$ para 5,5 h. O mesmo teor de grupos clorometila foi obtido quando a reação durou 5,5 h e um menor tempo de borbulhamento de $\mathrm{HCl}$ foi usado. Devido à grande influência sobre o grau de clorometilação dos copolímeros, esses parâmetros foram cuidadosamente estudados e os resultados obtidos estão resumidos na Tabela 4.

As reações CMDCE 1 a 5 foram realizadas com o objetivo de se estudar a influência da temperatura de reação sobre o grau de clorometilação dos copolímeros S-DVB, mantendose todos os demais parâmetros fixos. Observa-se uma diminuição do teor de grupos clorometila incorporados no copolímero com a diminuição da temperatura. Os copolímeros clorometilados nas reações CMDCE1 $\left(80^{\circ} \mathrm{C}\right)$ e CMDCE2 $\left(67^{\circ} \mathrm{C}\right)$ mostraram valores comparáveis para o teor de grupos clorometila, enquanto que o copolímero da reação CMDCE3 $\left(61^{\circ} \mathrm{C}\right)$ apresentou teor bem menor que os anteriores. Essa observação sugere que temperaturas em torno de $70{ }^{\circ} \mathrm{C}$ já são suficientes para o curso da reação de clorometilação. A reação CMDCE6 representa uma réplica da reação CMDCE1, e mostra, como era de esperar, uma reprodução do grau de clorometilação. Para garantir que a temperatura não tivesse influência sobre o estudo dos outros parâmetros, as demais reações foram realizadas a $80^{\circ} \mathrm{C}$ e a reação CMDCE1 (ou CMDCE6) foi considerada como padrão, a partir dela foram variados todos os parâmetros reacionais.

O estudo da razão entre a massa de copolímero e a massa de catalisador foi feito diminuindo-se a massa de $\mathrm{ZnCl}_{2}$ progressivamente e mantendo-se a massa de copolímero fixa (reações CMDCE6 a 9). Pode-se observar um máximo nos valores de teor de grupos $\mathrm{CH}_{2} \mathrm{Cl}$ quando $0,075 \mathrm{~g}$ de catalisador foi usado (7,5\% da massa do copolímero). Para $15 \%$ e $25 \%$ houve uma diminuição do teor de grupos incorporados. Este comportamento pode ser atribuído à formação de pontes metilênicas por meio da reação de alquilação de Friedel-Crafts entre um grupo clorometila já incorporado e um anel aromático. Esta reação lateral compete com a reação principal e leva a um aumento do grau de reticulação do copolímero, o que poderia ocasionar uma diminuição no grau de inchamento desses materiais. Entretanto, não houve variações significativas nesse parâmetro, isso pode significar que a extensão dessas 
Tabela 4. Condições reacionais e resultados para as reações de clorometilação de S-DVB em meio orgânico.

\begin{tabular}{|c|c|c|c|c|c|c|c|c|}
\hline Reação & IV & $\begin{array}{c}\mathrm{CH}_{2} \mathrm{Cl} \\
\left(\mathrm{mmol} \mathrm{g}^{-1}\right)\end{array}$ & $\mathrm{m} \mathrm{ZnCl}_{2}(\mathrm{~g})$ & $\mathrm{V}_{\substack{1,2-\mathrm{DCE} \\
(\mathbf{m L})}}$ & $\begin{array}{l}\mathbf{t}_{\text {reação }} / \mathbf{t} \mathbf{H C l} \\
\text { (h) }\end{array}$ & $\begin{array}{c}\mathbf{T} \\
\left({ }^{\circ} \mathbf{C}\right)\end{array}$ & $\left(\mathrm{g} \mathrm{cm}_{\mathrm{a}}^{-3}\right)$ & $\begin{array}{l}I_{\text {tol }} \\
(\%)\end{array}$ \\
\hline CMDCE1 & $(+)$ & 0,434 & \multirow{6}{*}{0,250} & \multirow{12}{*}{60} & \multirow{9}{*}{$3 / 3$} & 80 & 0,34 & 23 \\
\hline CMDCE2 & $(+)$ & 0,416 & & & & 67 & 0,32 & 23 \\
\hline CMDCE3 & $(+)$ & 0,182 & & & & 61 & 0,36 & 25 \\
\hline CMDCE4 & $(+)$ & 0,110 & & & & 53 & 0,31 & 20 \\
\hline CMDCE5 & $(+)$ & 0,0434 & & & & 41 & 0,30 & 23 \\
\hline CMDCE6 & $(+)$ & 0,0450 & & & & \multirow{10}{*}{80} & 0,29 & 21 \\
\hline CMDCE7 & $(+)$ & 0,489 & 0,150 & & & & 0,30 & 16 \\
\hline CMDCE8 & $(+)$ & 0,576 & 0,075 & & & & 0,31 & 27 \\
\hline CMDCE9 & $(+)$ & 0,254 & 0 & & & & 0,30 & 29 \\
\hline CMDCE10 & $(+)$ & 0,234 & \multirow{4}{*}{0,250} & & $4 / 4$ & & 0,30 & 14 \\
\hline CMDCE11 & $(+)$ & 0,409 & & & $2 / 2$ & & 0,31 & 35 \\
\hline CMDCE13 & $(+)$ & 0,312 & & & $3 / 1$ & & 0,35 & 21 \\
\hline CMDCE14 & $(+)$ & 0,450 & & \multirow{2}{*}{30} & \multirow{3}{*}{$3 / 3$} & & 0,32 & 16 \\
\hline CMDCE15 & $(+)$ & 0,518 & 0,125 & & & & 0,48 & 63 \\
\hline CMDCE16 & $(+)$ & 0,266 & 0,250 & 15 & & & 0,30 & 11 \\
\hline
\end{tabular}

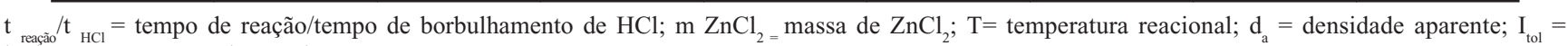
inchamento percentual em tolueno

reações foi baixa. O copolímero S-DVB- $\mathrm{CH}_{2} \mathrm{Cl}$, obtido em ausência de catalisador, apresentou um teor de grupos $\mathrm{CH}_{2} \mathrm{Cl}$ bastante significativo, embora mais baixo do que os obtidos em sua presença. Neste caso, a ocorrência da reação deve-se ao deslocamento do equilíbrio de formação do eletrófilo pela alta acidez do meio.

A formação do eletrófilo envolve, provavelmente, a protonação do oxigênio da carbonila aldeídica e o ataque do íon cloreto ao carbono deficiente de elétrons. O intermediário formado reage então com $\mathrm{ZnCl}_{2}$ formando um complexo catiônico capaz de ser atacado pela nuvem eletrônica dos anéis estirênicos. A reação lateral de formação de pontes metilênicas, uma segunda alquilação de Friedel-Crafts, é igualmente influenciada pela concentração do catalisador. Quando $15 \%$ e $25 \%$ de $\mathrm{ZnCl}_{2}$ foram usados, a reação de reticulação passou a ter papel significativo em comparação ao uso de 7,5\% de catalisador.

O tempo de reação também parece ser um fator que contribui para a formação de reticulações (pontes metilênicas). A reação realizada com $4 \mathrm{~h}$ de duração e borbulhamento constante de $\mathrm{HCl}(\mathrm{g})$ (CMDCE10) levou a um copolímero com grau de clorometilação bastante inferior ao obtido com $3 \mathrm{~h}$ de duração. $\mathrm{O}$ grau de inchamento em tolueno desse copolímero foi menor do que o clorometilado durante $3 \mathrm{~h}$, o que confirma a ocorrência da reação lateral. Comparando-se o efeito do tempo de reação e da razão copolímero/catalisador sobre a extensão da formação de pontes metilênicas, pode-se concluir que o tempo teve um efeito mais acentuado, pois a redução do teor de grupos clorometila e do grau de inchamento dos copolímeros foi muito maior quando o tempo de reação foi aumentado para $4 \mathrm{~h}$ do que quando a massa de catalisador foi aumentada. Provavelmente, após $4 \mathrm{~h}$ de reação, o consumo de reagentes atingiu o seu valor máximo e a reação entre os grupos clorometila e os anéis aromáticos passa a ser a principal do sistema. A reação realizada com $2 \mathrm{~h}$ de duração e borbulhamento constante de $\mathrm{HCl}(\mathrm{g})$ resultou em um copolímero com grau de clorometilação próximo ao apresentado pelos copolímeros clorometilados durante $3 \mathrm{~h}$. Entretanto, as reações realizadas com $3 \mathrm{~h}$ parecem ser mais completas.

Realizou-se uma reação com $3 \mathrm{~h}$ de duração e tempo de borbulhamento de $1 \mathrm{~h}$ (CMDCE13). O teor de grupos clorometila obtido nessa condição não foi muito inferior ao obtido com borbulhamento constante de $\mathrm{HCl}(\mathrm{g})$. Assim, podese supor que tempos maiores do que $3 \mathrm{~h}$ de reação sem borbulhamento constante de $\mathrm{HCl}(\mathrm{g})$ podem levar a graus de clorometilação comparáveis aos obtidos com $3 \mathrm{~h}$ e borbulhamento constante. Entretanto, um estudo mais detalhado deve ser feito para que sejam avaliadas essas relações.

A influência do volume de solvente usado na reação também foi avaliada. Segundo Pepper e colaboradores ${ }^{[17]}$, o aumento do volume de um solvente com afinidade pelo copolímero diminui a ocorrência da reação adicional de reticulação por meio de formação de pontes metilênicas. $\mathrm{O}$ decréscimo do volume de 1,2-DCE para $15 \mathrm{~mL}$ levou a um resultado que corrobora essa afirmativa, pois a reação 
CMDCE16 apresentou o menor teor de grupos clorometila incorporados no copolímero e também um baixo grau de inchamento em tolueno. Na presença de maiores volumes de 1,2-DCE (reações CMDCE1 e 14), o esqueleto polimérico está menos emaranhado devido à maior solvatação das cadeias, aumentando, assim, a distância entre os grupos clorometila e os anéis aromáticos e, conseqüentemente, dificultando a reação lateral de alquilação. $O$ grau de inchamento do copolímero obtido na reação CMDCE14 (30 mL de 1,2-DCE) também foi baixo, apesar de apresentar teor de grupos clorometila próximo ao obtido com a reação (CMDCE1). Neste caso, o grau de clorometilação atingido durante a reação CMDCE14 pode ter sido maior do que o determinado no material final. A formação posterior de pontes metilênicas pode ter diminuído o grau de clorometilação do copolímero final, resultando, assim, em um material com menor capacidade de inchar em tolueno. O mesmo fato explica os baixos valores de inchamento e do teor de $\mathrm{CH}_{2} \mathrm{Cl}$ no copolímero obtido pela reação CMDCE16.

\section{Conclusões}

A reação de clorometilação de copolímeros S-DVB usando-se a mistura $\mathrm{HCl}$ /formaldeído como agente clorometilante em presença de $\mathrm{ZnCl}_{2}$ como catalisador ocorre com maior conversão em meio orgânico. Em meio aquoso, são obtidos baixos graus de incorporação de grupos clorometila, mesmo nas condições mais drásticas. Este resultado pode ser explicado pela dificuldade de formação do eletrófilo em meio aquoso e pelo difícil acesso desse meio reacional ao interior das pérolas de copolímero.

Em meio orgânico, foram obtidos copolímeros com graus de clorometilação superiores aos obtidos em meio aquoso. A temperatura e a relação mássica entre o copolímero e o catalisador foram os parâmetros que mais influenciaram o grau clorometilação do copolímero. $\mathrm{O}$ aumento da temperatura acima de $67^{\circ} \mathrm{C}$ não mostrou melhora do rendimento da reação de clorometilação, enquanto que, acima de 7,5\% de catalisador, a ocorrência significativa de reação lateral começa a diminuir o teor de grupos clorometila. A diluição, ou seja, o aumento do grau de inchamento do copolímero no solvente, pode ajudar a prevenir as reações de reticulação adicionais.

A combinação dos parâmetros reacionais para a clorometilação de S-DVB em meio orgânico é fundamental para se controlar não somente o teor de grupos incorporados mas também a ocorrência de reticulações adicionais.

\section{Agradecimentos}

Os autores agradecem ao Conselho de Desenvolvimento
Científico e Tecnológico (CNPq) e à Fundação de Amparo à Pesquisa do Estado do Rio de Janeiro (FAPERJ) pelo apoio financeiro.

\section{Referências Bibliográficas}

1. Beauvais, R. A.\& Alexandratos, S. D. - React. Funct. Polym., 36, p.113 (1998)

2. Patel, S. A.; Sinha, S.; Mishra, A. N.; Kamath, B. V. \& Ram, R. N. - J. Mol. Catal., 192, p.53 (2003)3. Takeda, K.; Akiyama, M.; Kawakami, F. \& Sasaki - Bull. Chem. Soc. Jpn., 59, p.2225 (1986).

4. Feinberg, R. S. \& Merrifield, R. B. - Tetrahedron, 30, p. 3209 (1974).

5. Wright, M. E.; Toplikar, E. G. \& Svejda, S. A. Macromolecules, 24, p.5879 (1991).

6. Warshawsky, A. \& Deshe, A. - J. Polym. Sci.: Polym. Chem., 23, p.1839 (1985).

7. Olah, G. A.; Beal, D. A.; Yu, S. H. \& Olah, J. A.- Synthesis, p. 560 (1974).

8. Olah, G. A.; Beal, D. A. \& Olah, J. A. - J. Org. Chem., 41 (9), p.1627 (1976).

9. DeHaan, F. P.; Djaputra, M.; Grinstaff, M. W.; Kaufman, C. R.; Keithly, J. C.; Kumar, A.; Kuwayama, M. K.; Macknet, K. D.; Na, J.; Patel, B. R.; Pinkerton, M. J.; Tidwell, J. H. \& Villahermosa, R. M. - J. Org. Chem., 62, p.2694 (1997)

10. Ye, C.; Marks, T. J.; Yang, J.; Wong, G. K. Macromolecules, 20, p.2322 (1987).

11. Itsuno, S.; Uchikoshi, K. \& Ito, K. - J. Am. Chem. Soc., 112, p.8187 (1990).

12. Avram, E. \& Luca, C. - Polym.-Plast. Technol. Eng., 35 (5), p. 757 (1996).

13. Dalal, M. \& Ram, R. N. - Eur. Polym. J., 33 (9), p. 1495 (1997).

14. Dalal, M. \& Ram, R. N. - J. Mol. Catal. A: Chemical, 159, p. 285 (2000).

15. Fuson, R. C. \& McKeever, C. H. - "Chloromethylation of Aromatic Compounds", in: Organic Reactions, vol. 1, cap. 3, Roger Adams (ed.), John Wiley \& Sons, Inc., New York (1942).

16. Guyot, A.; Hodge, p.; Sherrington, D. C. \& Widdecke, H. - React. Polym., 16, p.233 (1991/1992).

17. Pepper, K. W.; Paisley, H. M. \& Young, M. A. - J. Chem. Soc, p.4097 (1953).

Enviado: 22/06/04

Aprovado: 20/09/04 